\title{
DATA ACQUisition AS A Design PARAMETER In THE MODERN AEM
}

\section{Nestor J. Zaluzec}

Electron Microscopy Center for Materials Research, Materials Science Division, Argonne National Laboratory, Argonne, Illinois 60439, USA

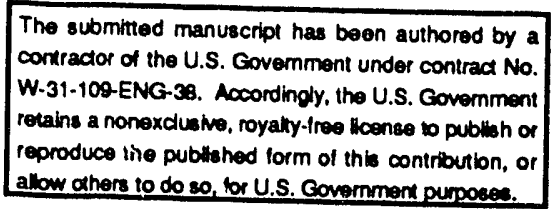

April 1993

\section{DISCLAIMER}

\begin{abstract}
This report was prepared as an account of work sponsored by an agency of the United State
Government. Neither the United States Government employees, makes any warranty, express or implied nor any agency th. reof, nor any of their bility for the accuracy, completeness, or usefulied, or assumes any legal liability or responsiprocess disclosed, or represents that its use would of any information, apparatus, product, or ence herein to any specific commercial product not infringe privately owned rights. Refermanufacturer, or otherwise does not product, process, or service by trade name, trademark, mendation, or favoring by the United States constitute or imply its endorsement, recomand opinions of authors expressed herein United States Government or any agency thereof.
\end{abstract}

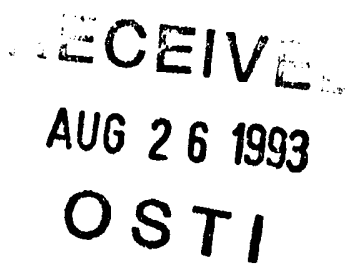

Work supported by the U.S. Department of Energy, BES-Materials Sciences, under Contract No. W-31-109-Eng-38.

Contributed manuscript submitted to the Electron Microscopy Society of America 51st Annual Meeting, Cincinnati, Ohio, August 9-13, 1993. 


\title{
DAta ACQuisition as A Design PARAMETER in The MOdern AEM
}

\author{
Nestor J. Zaluzec
}

\section{Electron Microscopy Center for Materials Research, Materials Science Division, Argonne National Laboratory, Argonne, Illinois 60439 , USA}

Data is the essential component of all scientific investigations. From this information we, as experimentalists, formulate ideas, test theories, derive conclusions and design new experiments to continue our explorations of materials both in the physical and life sciences. Clearly without it we would be left with literally nothing to do. With that thought in mind it should be intuitively obvious that there are two essential aspects of all studies. The first is a well formulated experiment whose results will provide relevant data and second that the methodology/instrument we choose to utilize to collect the data has the ability to acquire the information we need to obtain about an object or phenomenon. By extension one would assume that having chosen an instrument that it was designed with the thought in mind to maximize the amount and quality of information for which it is being employed.

We can break down any experimental system into the following areas: the environment and manipulation of the specimen in the instrument, the probe used to investigate the specimen, the detectors used to measure information, and finally the transfer of data from the experiment to the outside world. It is essential that any experiment be considered to be a system composed of mutually complementary parts all of which should be working in concert to provide the maximum amount of data with a reasonable effort. The bane of the analyst, especially in the field of microscopy, is the fact that, more often than not, the configuration of the analytical equipment which we use for our experiment(s) is not that of a complete system but rather a collection of subsystems which have not been optimized. Optimizing each of these is not a trivial endeavor and if one considers the task of integrating each into an operating system then the job can become a nightmare. This is particularly true when the various components have been manufactured by different individuals/organizations who may not have been closely coordinated together. This is notably relevant in the modern analytical electron microscope (AEM). At the risk of alienating most every manufacturer let us present a pessimistic view of an AEM system.

Microscope manufacturers tend to concentrate their considerations to electron optics and seek to maximize their capabilities in imaging and probe forming systems. A few give some extra consideration to the specimen area, but most favor speed of specimen exchange and ease of operation over the optimization of the environment. This is a direct consequence of the fact that the primary data to be generated is an electron scattering experiment resulting in an image and/or diffraction pattern. Data output is generally recorded on film with high spatial resolution, but relatively small linear dynamic range and data transfer rates are extremely slow $(<1 \mathrm{kHz})$. Some attempts are being made to implement digital image acquisition using electron sensitive camera systems however, a good fraction of the work is being done by accessory manufacturers. Control of the electron dose in the basic instrument is visual, and absolute measurements of the incident and scattered electrons are seldom conducted. Ancillary detector manufacturers (XEDS, EELS, AES, CCD's....) while attempting to maximize the performance of their respective spectroscopic systems are usually limited by the constraints (in the positioning and size of their respective detectors) placed upon them by the instrument manufacturer. But then again few of these have these have tried to suggest other than minor changes to the basic system. Data throughputs from the detector to the analyzer vary wildly ranging from $<1 \mathrm{kHz}$ on XEDS systems to $>10 \mathrm{MHz}$ on electron counting systems. Furthermore, once the data is measured in a given acquisition system transfer of this information to an alternate analysis system can vary in speed by orders of magnitude. Control of some instruments ranges from almost Babalonic in nature: antiquated, confused and atrociously slow $(<1 \mathrm{kHz})$; to some of the newer 
systems that are so tightly integrated with flashy workstations that it is nearly impossible to modify them. Finally inter-system communication between the various data acquisition, control and analysis systems is almost nonexistent when the subsystems come from different sources.

Many of the above pessimistic viewpoints/compromises are based upon the driving forces in the market place as the majority of analysts do not need to deal with pushing the frontiers of analysis. State-of-the-art may mean the latest commercial product, and if the product generates the appropriate data and answers the questions posed then it was a suitable instrument and the most cost effective solution to our first question posed.

On the other hand the answers needed may require experimental configurations which push the limits of data collection. In this scenario, given the time (and money) one can attempt to optimize an analytical system by first considering the fact that data collection is of prime importance and it should dictate the design of an instrument. Such an instrument is then a resource for the community as a whole and serves to define the next generation of instrument available to the average user. The question then to ask is: as a system how do we design the "instrument" to generate, acquire, store and transmit the current generation of microanalytical data and in addition keep sufficient versatility to expand to experimental configurations yet to be implemented. The points to consider are:

- Maximize the available space about the specimen to allow multiple Hetector configurations and reasonable expansion for future developments yet to be "discovered" within the lifetime of the instrument ( $\sim 10-15$ years).

- Configure the specimen manipulation systems to allow maximal manipulation of the sample and the design of new stages.

- Achieve an environment which is free of contamination during the course of an experiment.

- Develop (spectroscopic) detector systems with data input rates which can keep up with the generation rates created by the all probes being employed and free of "system peaks" from the immediate surroundings. (This is a major obstacle particularly in XEDS!).

- Establishment of well defined, controlled and stable high intensity probes with measurement systems capable of characterizing the absolute probe characteristics to better than $0.1 \%$.

- Implement high speed digital control of instrument with average $\mathrm{J} / \mathrm{O}$ rates $>500 \mathrm{kbyte} / \mathrm{sec}$, over all system parameters/functions. Digital counting systems with data depths of at least 24 bits and digital scanning systems capable of up to $4 \mathrm{Kx} 4 \mathrm{~K}$ area scans. Configurations should implement multiple microprocessors with local data storage to direct different tasks and have multiple $\mathrm{V} / \mathrm{O}$ links including high speed serial, SCSI and DMA buses for instrument-to-instrument communication. Basic instrument interface controls must be developed to allow interactive control of the entire system by ancillary equipment using a standard command line interface.

- High speed data transfer $(>10 \mathrm{Mb} / \mathrm{sec})$ from the primary instrument to ancillary systems using established international protocols (TCP/IP) and data formats (MSA/MAS file formats, TIFF imaging.....).

- Local data storage ( $>0.5-2 \mathrm{~Gb})$ on data acquisition systems for short term analytical imaging.

- Remote workstations for data analysis.

Most of these items can be implemented today as they within the technological reach of present day instrumentation. It only remains for the community as a whole to demand this of the manufacturers.

This work was supported by US. DoE under contract BES-MS W-31-109-Eng-38. 

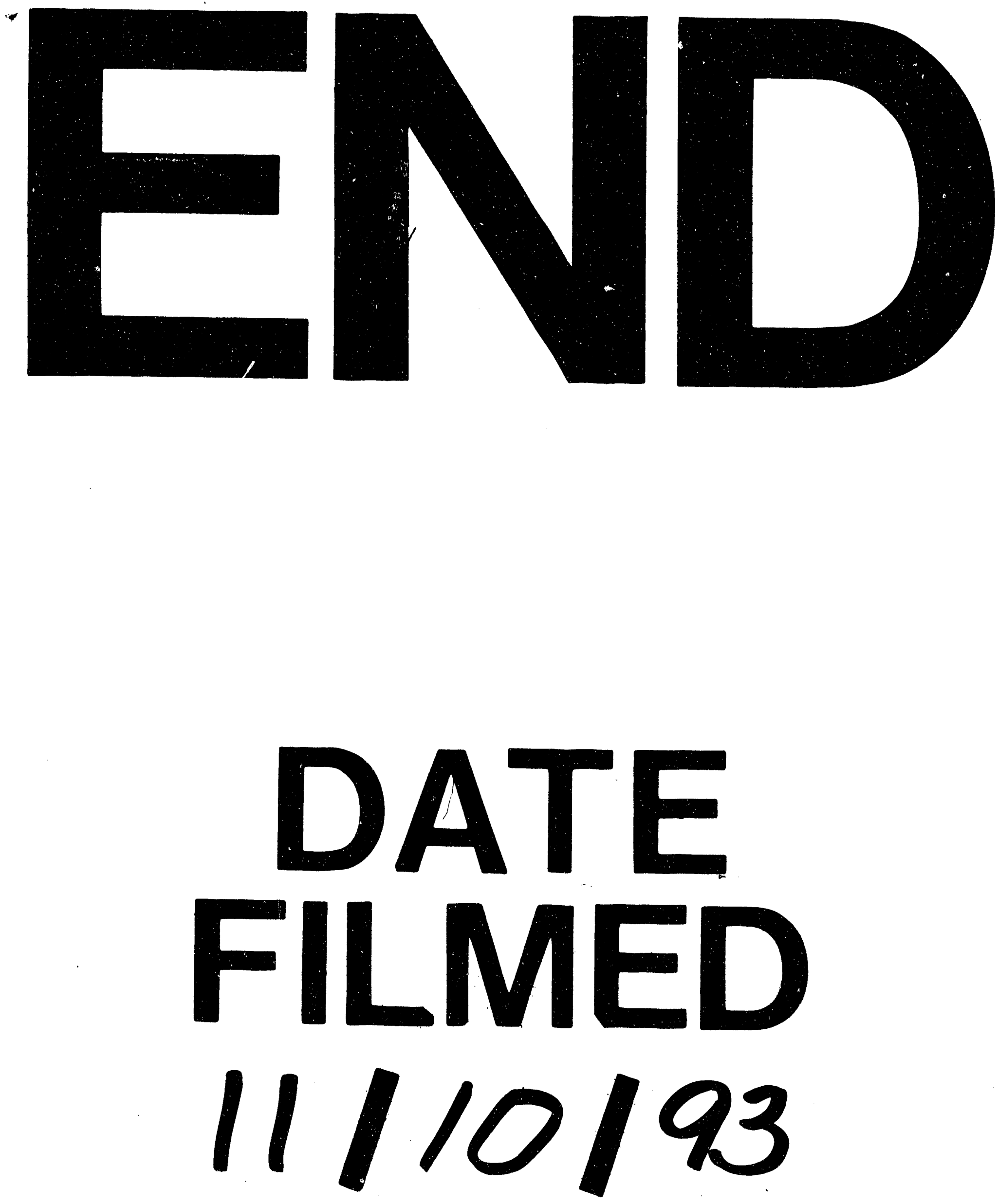
